\title{
ARTIFICIAL INTELLIGENCE IN MILITARY APPLICATION - OPPORTUNITIES AND CHALLENGES
}

\author{
István SZABADFÖLDI \\ National University of Public Service, Budapest, Hungary \\ szabadfoldi.istvan@uni-nke.hu
}

\begin{abstract}
Artificial Intelligence (AI) is playing an increasing role in planning and supporting military operations and becoming a key tool in intelligence and analysis of the enemy's intelligence. Another field of application of AI is the field of application of autonomous weapon systems and vehicles. The use of AI is expected to have a greater impact on the military functions of human-machine interfaces (machine-learning, man-machine teaming). AI promises to get over the " $3 V$ challenge" (volume, variety and velocity) of Big Data, and is also expected to reduce the risks concerning the other " $2 V$ " (veracity, value), and to render data processing on a controlled level of decision based on AI's knowledge. The aim of the article is to provide an overview on the potentials of application of AI in the military and to highlight the need to identify and define measurable indicators to evaluate benefits of state-of-the-art technologies and solutions which are expected to improve quality and performance of operations focusing on key areas as of situational awareness and decision-making support and also logistic and operational planning as well as modelling and simulation (M\&S).
\end{abstract}

KEYWORDS: artificial intelligence, big data, analytics, autonomous systems, machine learning

\section{Introduction}

Since the beginnings of the mid 1900s, the scientific field of Artificial Intelligence (AI) went through periods of intense development and drawbacks. From year 2000, however, a significant and persistent growth in AI research, development, and application can be perceived. AI research began to speed up around 2001, and then commercial products and services started permeating the market in large numbers especially since early 2010s. Within roughly a decade, AI has grown up out of the laboratories and penetrated the economy from industrial design, through manufacturing, as well as to the consumer's services (NATO Parliamentary Assembly, 2019).

The observers and experts forecast high global economic impacts associated with the application, development, and adoption of AI over the next ten years, and a reasonable estimation put the range of AI's economic impact to be between $\$ 1.49$ trillion and $\$ 2.95$ trillion in this period (Chen et al, 2016). 
The main objective of the article is to survey and analyze the course of development of AI, and provide an overview of the current status of applicability and expectations in the military domain. The method of research was surveying and comparison of publications and policy papers of the NATO and the EU, analysis of forecasts and reports of AI state-of-the-art techniques, and presenting opinions of $\mathrm{AI}$ commercial and military solution providers.

\section{Research Methodology}

The research methodology for the topic was based on a review of the relevant scientific literature and interviews with military personnel. It is important to note and understand that the access to available sources is limited in details - especially concerning the technical and functional implementation of the required features and capabilities - by the nature of the relative novelty and confidential sensitivity of the technologies. The methodology is there for limited to open access sources and materials.

\section{What Is Artificial Intelligence - Overview and Demystification}

Artificial Intelligence, although a term used since the 1950s, has still not a generally accepted definition today. AI cannot be interpreted as a stand-alone application, but as a technology that supports existing functional applications and is eventually based on algorithms designed to solve specific problems, collecting, organizing, processing, analyzing, transmitting, and responding to larger data sets, suitable and capable of corresponding to the cognitive ability of the human intellect, and operations approaching it.

There are basically three types of AI:

- Narrow Artificial Intelligence (Narrow AI). Narrow artificial intelligence, also called "weak AI", is a computer system that can perform a narrowly defined task more efficiently than a human. That is where most of the AI applications extend today.

- General Artificial Intelligence (General AI), sometimes referred to as "Strong AI", can outperform human achievements in any intellectual task. For example, we can see robots with this type of AI in movies where they act according to their own goals based on conscious thoughts.

- Artificial Super Intelligence (ASI) is expected to be able to outperform humans in almost all areas, but especially in scientific creativity, logic and wisdom, and also in social skills.

Many scientists have a strong doubt that this last would ever be achievable at all. Furthermore, even if the ASI or close to that level of AI will be reached, the human contribution to the process of AI application at some point remains a must, since ethical, intuitional and especially spiritual motives and capabilities are exclusively human characteristics, even if, to some extent, machines can emulate them.

A subset of MI is machine learning (ML), which teaches computers with mathematical data models with or without direct supervision. Machine learning uses algorithms to identify patterns in the data creating a data model to provide predictions and responses. The advanced level of ML is Reinforcement Learning when the system is reinforced with positive feedbacks in the recognitions.

In the case of Deep Learning, the machine is taught with complex amounts of data for complex tasks with the help of neural networks created by the analogy of the human brain, in which the neurons perform a sub-function or summarize them. Here it is important to mention the so-called Black Box phenomenon, in which the 
human being is no longer able to follow the process taking place in the individual neuronal levels. So, they pose a significant reliability risk.

There are increasing number of publications and public events on AI, nevertheless or rather because of these, widespread myths and misconceptions remain about what $\mathrm{AI}$ really is and what $\mathrm{AI}$ systems can do. These misrepresentations make it difficult and unproductive to understand and discuss the opportunities and risks of AI in general and in the security field in particular (Saalman, 2019).

The possibilities and limitations of AI technology have to be clearly understood and considered especially for decision makers to avoid creating illusions and launching projects with hardly achievable targets.

\section{Emerging and Disrupting Technologies (Edts)}

Before AI is discussed in more details it is important to put it in a wider context of other technologies which are highly interrelated scientific areas and are considered as major strategic influencers over the next 20-years. Some of these areas are momentarily in early stages of development but are expected to go through rapid development. These EDTs are: Big Data, Artificial Intelligence (AI), Autonomous Vehicles, Space, Hypersonic Aerial Vehicles, Quantum technology, Biotechnology, Novel Materials (NATO Science \& Technology Organization, 2020). These EDTs are expected to make comprehensive societal impacts, infiltrating in telecommunications, entertainment, medical sciences, human-computer interactions, financial services, agriculture, transport industry, industrial manufacturing and public security and safety as well.
The democratization of technology raises the security concern because of the reduced costs and ease of access. There is a need of higher level of regulation to protect the society from technologically advanced malicious actors who may be capable to produce highly threatening equipment and solutions based upon easily available knowledge. Although Chemical, Biological, Radiological and Nuclear technologies (CBRN) are generally highly regulated, however these regulations may be inadequate if "anyone can set up a bioengineering laboratory in a backyard or basement" (NATO Science \& Technology Organization, 2020, p. 35).

\section{AI Development and Application in Military}

\subsection{The Course of Development of AI}

AI went through three development stages since the concept came to existence somewhat seventy years ago (Figure no. 1). In the first stage the solutions focused on rules-based approaches like decision trees, Boolean and fuzzy logic and were generally called expert systems. In the second stage AI developers focused on the development and application of statistical methods which resulted in the concept and methodology of machine learning. These were successfully coming up with solutions such as e-mail spam filtering and internet search engines. The third stage of development, which is going on now, introduced the use of human-like learning methods such as neural networks, defining the concept and technology of deep learning, and proved to be successful in sensing and perception (NATO Science \& Technology Organization, 2020). 


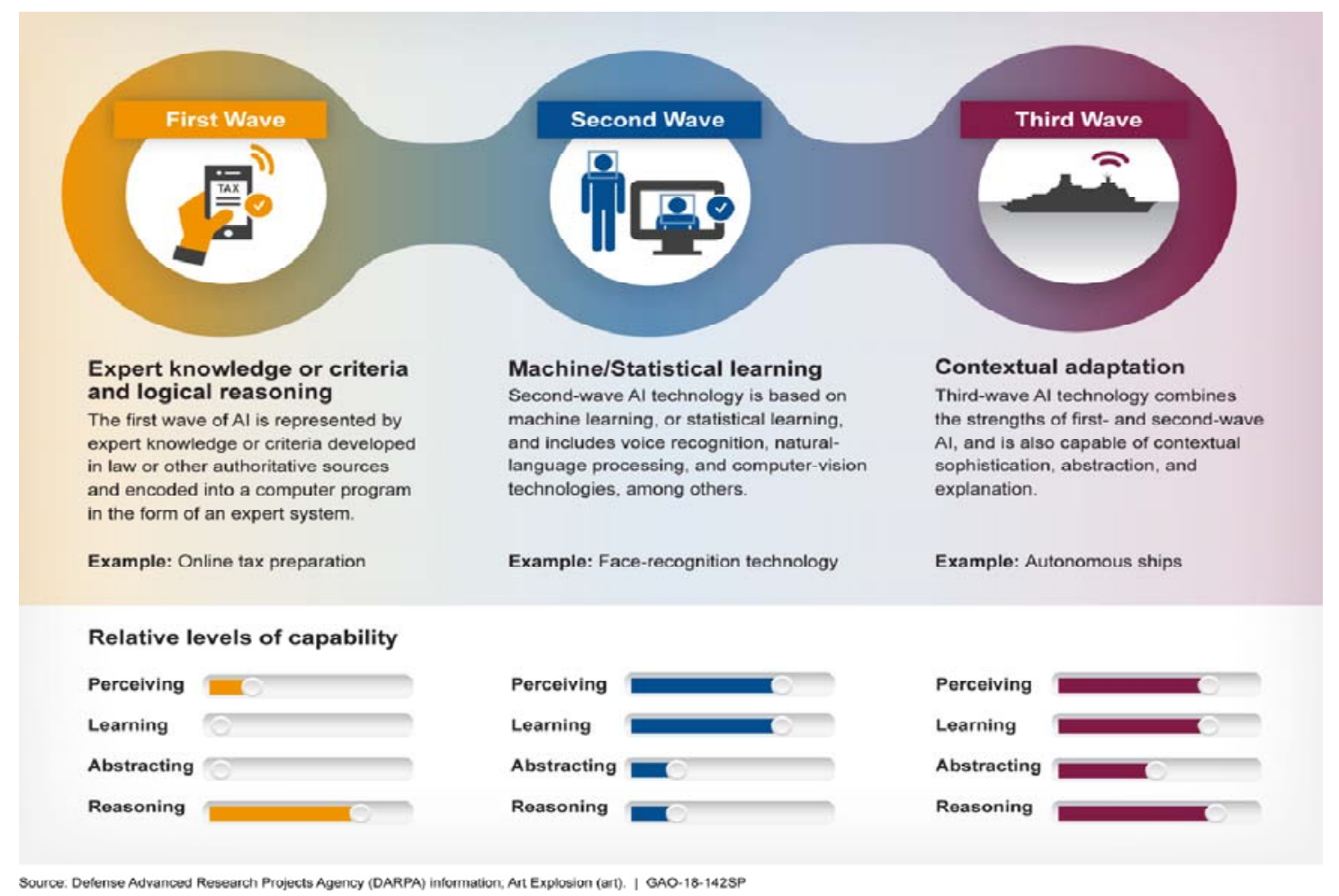

Figure no. 1: The course of development of AI

(Source: United States Government Accountability Office (GAO), 2018)

The Gartner Hype Cycle for AI represents the expected course pf development and application of AI

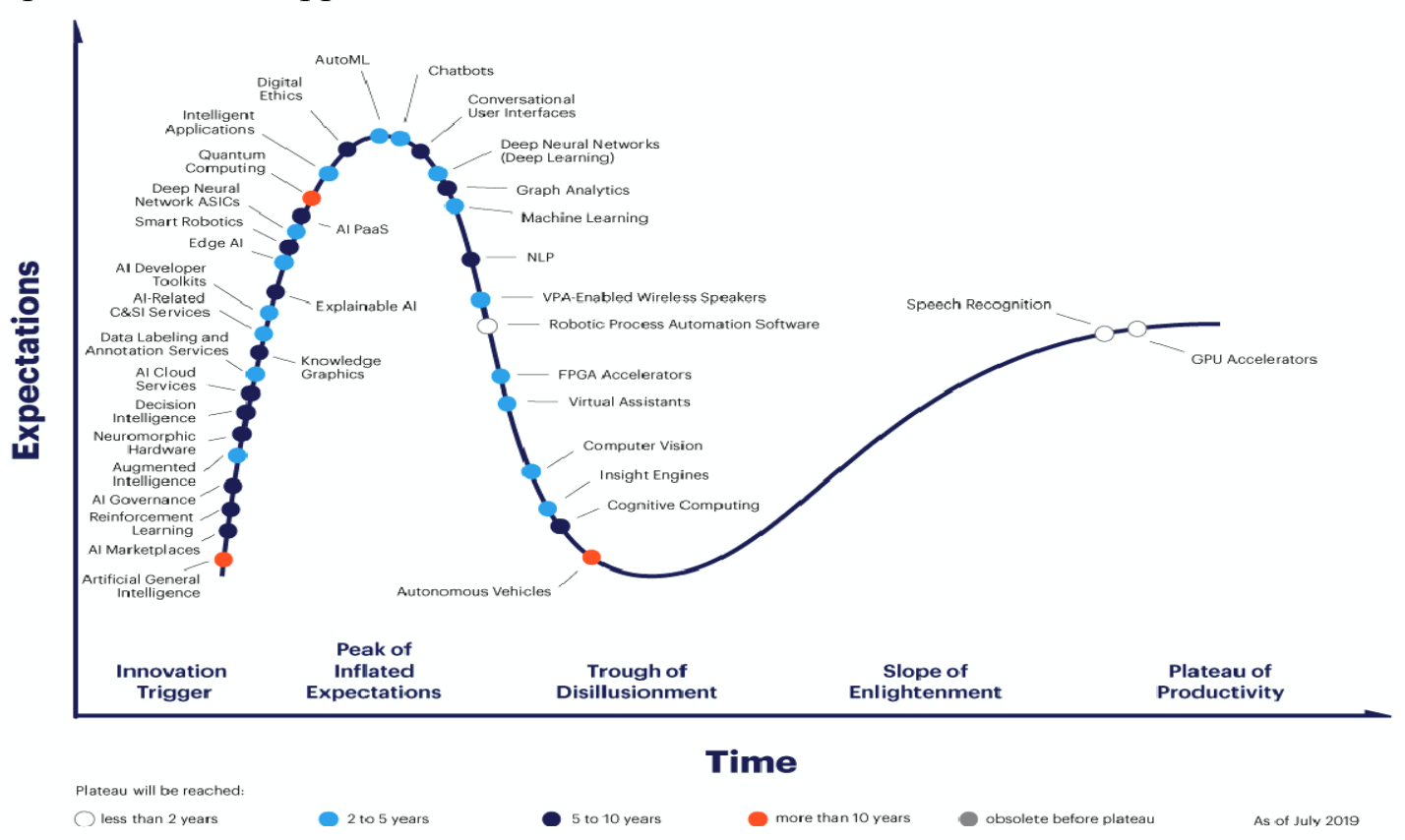

Figure no. 2: Gartner Hype Cycle for AI

(Source: Gartner, 2019) 
Deep-learning methods are expected to be further developed, besides, new research areas including neuromorphic computing are raising, emulating the neural structure and operation of the human brain. Together with these, adversarial machine learning techniques are being developed to understand how to confuse enemy's AI systems. So basically, as of today the defense of integrity of AI systems is becoming a critical challenge parallel with the proactive application.

\subsection{Expectations for AI in Military}

As for the expectations concerning AI in military application over the next decade, some of its techniques will define or re-define key advanced military technologies.

AI solutions will be first of all intelligent, exploiting integration of AI knowledge-focused analytic capabilities. Then the AI solutions will be interconnected to utilize the network of virtual and physical domains, including sensors, organizations, individuals and autonomous agents, also exploiting the advantage of blockchain technology for data integrity. They will be distributed to utilize decentralized and ubiquitous largescale sensor networks, storage, and computation. And last but not least, they will be digitally intermingling human, physical and information domains to support novel disruptive effects (NATO Science \& Technology Organization, 2020).

\subsection{Key Areas to Examine of AI Application Advances}

"The Pentagon is figuring ways to harness artificial intelligence (AI) for advantages as far-flung as battlespace autonomy, intelligence analysis, record tracking, predictive maintenance and military medicine. AI is a key growth investment area for DoD, with nearly $\$ 1$ billion allocated in the 2020 budget. The Defense Department's Joint Artificial
Intelligence Center (JAIC) will see its budget double to over \$ 208 million, with significant increases likely in 2021 and beyond ... The military is currently seeking to integrate AI into weapon systems development, augment human operators with AI-driven robotic maneuver on the battlefield and enhance the precision of military fires" (DoD Growth in Artificial Intelligence: The Frontline of a New Age in Defense, 2019).

AI already has deep impacts on nuclear armament, cyber and information warfare, materials and bio-technologies, aerospace, and considered that these effects will have the similar result on global world order that the appearance of nuclear weapons created. The AI systems are also expected to bring about an adversarial AI arms-race.

Machine learning uses mathematical algorithms and formulas to extract patterns from masses of data. However, if an adversary can see enough of the inputs and the outputs of our AI system, they can deduce what algorithms are operating, just as reverse engineering works. The whole game may be turning into a battle between adversarial groups of mathematicians, similarly to the codebreaking competition struggle of World War II and the Cold War (Artificial Intelligence the Frontline of a New Age in Defense, n.d.).

Let's examine some specific AI military applications in important domains where the revolutionary effects are expected and can already be perceived in leading military powers.

In case of C4ISR (Command, Control, Communications, Computers, Intelligence, Surveillance and Reconnaissance), the combatting units use trusted AI-enabled autonomous systems that are capable of carrying out tasks that are deemed "dull, dirty, dangerous or dear" and provide AI-enabled decision support to warscenarios and AI-recommended courses of action (COA). AI supported data fusion, 
categorization and targeting provide intelligence analysts higher capability of TCPED (tasking, collecting, processing, exploiting, disseminating) and retrieving information. AI integration into C4ISR process results better indications and warning, information and knowledge management tools, resulting more reliable intelligence analyses.

Autonomous systems, UxVs (Unmanned Vehicles like UAV, UGV, AUV etc.) can be operating on a much higher efficiency and safety level with AI support. The implementation of deep learning systems into unmanned platforms will significantly extend robotic capabilities for navigation.

AI supports long term Capability Planning by the development of analytical solutions, including supporting complex decision-making by assessments of complex factors.

AI technology improves autonomy to achieve sensor integration and data fusion to satisfy requirements for fast and reliable detection, identification, and monitoring (DIM) of CBRN hazards.

\subsection{Military Uses of Artificial} Intelligence, Automation, and Robotics (MUAAR) Project

Having realized the importance of technological lead of AI development and application, the NATO launched the Military Uses of Artificial Intelligence, Automation, and Robotics (MUAAR) project under the Multinational Capability Development Campaign (MCDC) in 2020. The project's scope was to develop concepts and capabilities to address the challenges of conducting joint coalition operations and provide assessments on them.

The project examined areas of the logistics, integrated air \& missile defense, the electromagnetic spectrum, the space, cyberspace, air, land and maritime domains. The project made assessment on the obstacles to implementation/integration of AA\&R capabilities and strategies to overcome hindrances. The project objective aimed to provide assessment of present and future military tasks and functions that could benefit from AA\&R considering also paybacks in efficiency and cost-savings (NATO Allied Command Transformation Operational Experimentation, 2020).

\section{Interrelation of AI and Big Data Advanced Analytics (BDAA) in Defense \\ Artificial Intelligence is strongly connected and interrelated to Big Data technology. Data feed for efficient AI application is a key success factor and the data feed has a wide variety of sources. Therefore, Big Data technological development has key impact on AI solutions and vice versa.}

Big Data technology accomplishes data processing and analysis that brings forth significant volume, velocity, variety, veracity and visualization challenges. Digitalization, spreading of sensor technology, IoT and virtualization of social media played significant role in the development of Big Data.

\subsection{Expected Impact of AI and $B D A A$ in Defense \\ Big Data Advanced Analytics} encompasses analytical methods for understanding and visualizing masses of information.

BDAA has four basic components:

- collection (sensors);

- communication;

- analysis;

- decision making (NATO Science

\& Technology Organization, 2020). 


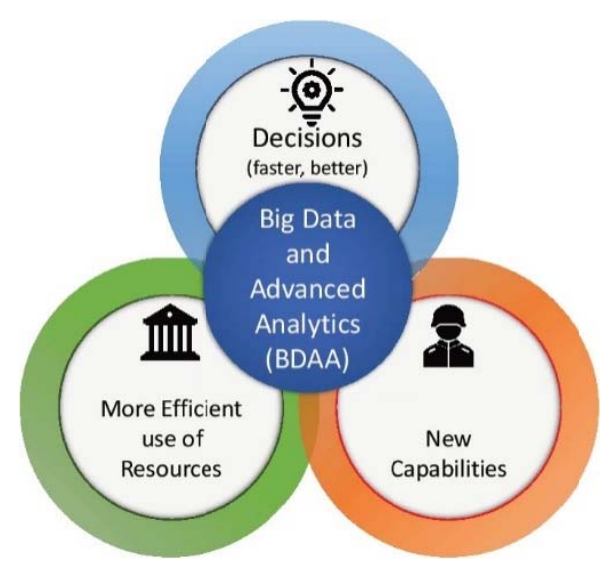

Figure no. 3: BDAA Goals

(Source: NATO Science \& Technology Organization, 2020)

Sensors are key for collecting data and they are coming from smart textiles, over-the-horizon (OTH) and passive radar systems, quantum sensing, Digital Twins (detailed virtual models of a weapon system), Computational Imaging (CI), and Microwave photonics delivering higher performance together with lower power, providing robust sensing capabilities and wireless communication on the battlefield (NATO Allied Command Transformation Operational Experimentation, 2020).

Figure no. 4 illustrates the interrelation of AI, ML and BDAA.

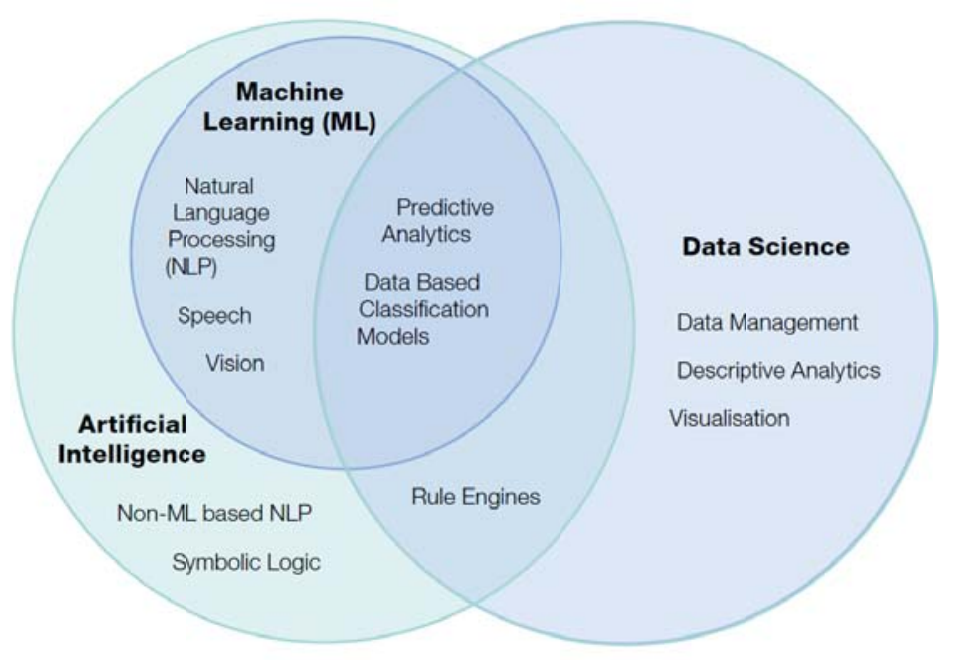

Figure no. 4: The Relationship between AI, Machine Learning and Data Science (Source: Torres, Hart, \& Emery, 2019)

\subsection{BDAA Impacts on Specific Military Areas \\ With the rapid development and cost-} efficient access to sensors of high sensitivity and the use of autonomous systems, raising the ability to detect and classify threats across physical and virtual operational domains, BDAA will result in more efficient ISR and is expected to provide higher level Situational Awareness (SA) by advanced mapping of mission areas for both planning and preparation. It will have increasing impact in Training and Readiness, and will result in more 
efficient Enterprise Management of the organizations, optimizing business processes, as well as real-time monitoring of the results of decisions. BDAA can provide predictive assessments through advanced modeling and simulation. Logistics will benefit from BDAA as well because of the increased integration of the sensors monitoring the weapon systems. Real-time inventory monitoring can also be achieved and the use of digital twins increases the effectiveness of the logistic system.

BDAA has key role in Support of Operations via large quantities of sensor data, and combining with AI, it provides a comprehensive approach to operational planning (OPLAN), courses of actions analysis and targeting.

\section{Critical Challenges of AI in Areas of Research}

In order to achieve and fully exploit the opportunities and capabilities mentioned above, Artificial Intelligence needs further advances in the following areas.

One of them is solving the Black Box Problem. Black box can be a device or system that allows seeing the input and output, but does not provide view of the internal processes. The AI Black Box means that in many AI-based tools the algorithm processing sections and nodes which are sequentially connected to refine data and results are not transparent in terms of exact input and output. This is especially true in case of Deep learning utilizing a large artificial neural network, with many hidden layers, and it 'learns' on its own by recognizing patterns layer by layer.

The Explainable AI is expected to be the solution for Black Box problem, as an AI tool that will provide transparent results on those nodes of layers (Zednik, 2019).

That functionality is not yet available in each solution, so the Black Box problem remains a significant risk to which the EU EDIDP called the attention to avoid in submitting proposals to be funded by EU EDF fund (European Commission, 2020).

Further research is necessary in the areas of Human-Machine Teaming, since human involvement in military operations remain no matter how advanced the AI techniques are. Machine and deep learning, adversarial AI, and neuromorphic and probabilistic computing in order to achieve advanced algorithms are also areas requiring further research to provide effective algorithms.

Since AI is a technology that can raise the efficiency of predictive and cognitive data analytics, the need for processes to transform both structured and unstructured data into insights for decisionmakers is key for applicability. Structured data can be processed by machine learning techniques by neural networks, while unstructured data is processed by using deep learning and natural language technics.

These tools may be applied to achieve more realistic analysis and personalized training within modeling and simulation, adaptive techniques in electronic warfare and development of AI agents for defensive and offensive cyber operations throughout the entire Infospace. The information gained through AI can be weaponized and hybridwarfare countermeasures can be achieved.

\section{Conclusions}

AI is definitely opening new perspectives in defense technologies. There are high expectations concerning the application of AI techniques in several military domains; however there are still hindering factors and unsolved issues for further research in order to fulfill those expectations.

As it was put by Breaking Defense: "what we call artificial stupidity: the ways algorithms can misinterpret the world in ways no human ever would, because they interpret data in terms of mathematics and logic without instinct, intuition, or common sense. It turns out such artificial stupidity is 
something you can artificially induce. The most famous example is an experiment in which strategically applied reflective tape caused the AIs used in self-driving cars to misclassify a STOP sign as a speed limit" (Artificial Intelligence the Frontline of a New Age in Defense, n.d., p. 17). It does not mean, however, that we have to be sceptic concerning AI widespread applicability, but rather understand that more intellectual effort and money should be poured into the $R \& D$.

\section{REFERENCES}

Artificial Intelligence the Frontline of a New Age in Defense. (n.d.). Breaking Defence, available at: https://cdn2.hubspot.net/hubfs/2097098/MCM120_BreakingDefense_AI ebook R1\%20(1).pdf, accessed on 29 March 2021.

Chen, N., Christensen, L., Gallagher, K., Mate, R., \& Rafert, G. (2016). Global Economic Impacts Associated with Artificial Intelligence, 23.

DoD Growth in Artificial Intelligence: The Frontline of a New Age in Defense. (2019). Breaking Defence, available at: https://breakingdefense.com/2019/09/dod-growth-in-artificialintelligence-the-frontline-of-a-new-age-in-defense/, accessed on 09 April 2021.

European Commission. (2020). European Defence Industrial Development Programme (EDIDP), available at: https://ec.europa.eu/research/participants/data/ref/other_eu prog/ edidp/wp-call/edidp_call-texts-2020 en.pdf, accessed on 09 April 2021.

Gartner. (2019). Gartner Hype Cycle for Artificial Intelligence.

NATO Parliamentary Assembly. (2019). Artificial Intelligence: Implications for NATO Armed Forces. NATO PA 149 STCTTS 19 E rev.1 fin, Science and Technology Committee.

NATO Allied Command Transformation Operational Experimentation. (2020). Military Uses of Artificial Intelligence, Automation, and Robotics (MUAAR), available at: https://www.act.nato.int/application/files/5515/8257/4725/2020 mcdc-muaar.pdf, accessed on 19 March 2021.

NATO Science \& Technology Organization. (2020). Science \& Technology Trends 2020-2040. Brussels, Belgium, available at: https://www.nato.int/nato static fl2014/ assets/pdf/2020/4/pdf/190422-ST_Tech_Trends_Report_2020-2040.pdf, accessed on 19 March 2021.

Saalman, L. (2019). The Impact of Artificial Intelligence on Strategic Stability and Nuclear Risk. Vol. II, Sweden: Stockholm International Peace Research Institute.

Torres, T.M., Hart, G., \& Emery, T. (2019). The Dstl Biscuit Book Artificial Intelligence, Data Science and (mostly) Machine Learning. $1^{\text {st }}$ edition revised, available at: https://assets.publishing.service.gov.uk/government/uploads/system/uploads/attachment data/ file/850129/The Dstl Biscuit Book WEB.pdf

United States Government Accountability Office (GAO). (2018). Report to the Committee on Science, Space, and Technology, House of Representatives. GAO-18-142SP, available at: https://www.gao.gov/assets/gao-18-142sp.pdf

Zednik, C. (2019). Solving the Black Box Problem: A Normative Framework for Explainable Artificial Intelligence, available at: https://arxiv.org/ftp/arxiv/papers/ 1903/1903.04361.pdf, accessed on 06 April 2021. 